\title{
Article \\ Genome-Wide Identification and Transcriptional Analysis of Arabidopsis DUF506 Gene Family
}

\author{
Sheng Ying ${ }^{\dagger}$
}

check for

updates

Citation: Ying, S. Genome-Wide Identification and Transcriptional Analysis of Arabidopsis DUF506 Gene Family. Int. J. Mol. Sci. 2021, 22, 11442. https://doi.org10.3390/ ijms222111442

Academic Editor: Bartolome Sabater

Received: 16 September 2021

Accepted: 21 October 2021

Published: 23 October 202

Publisher's Note: MDPI stays neutral with regard to jurisdictional claims in published maps and institutional affiliations.

Copyright: (C) 2021 by the author. Licensee MDPI, Basel, Switzerland. This article is an open access article distributed under the terms and conditions of the Creative Commons Attribution (CC BY) license (https:// creativecommons.org/licenses/by/ $4.0 /)$
Noble Research Institute LLC, Ardmore, OK 73401, USA; yingshen@msu.edu

+ Current address: Department of Biochemistry and Molecular Biology, Michigan State University, East Lansing, MI 48823, USA.

\begin{abstract}
The Domain of unknown function 506 (DUF506) family, which belongs to the PD-(D/E)XK nuclease superfamily, has not been functionally characterized. In this study, 266 DUF506 domaincontaining genes were identified from algae, mosses, and land plants showing their wide occurrence in photosynthetic organisms. Bioinformatics analysis identified 211 high-confidence DUF506 genes across 17 representative land plant species. Phylogenetic modeling classified three groups of plant DUF506 genes that suggested functional preservation among the groups based on conserved gene structure and motifs. Gene duplication and $\mathrm{Ka} / \mathrm{Ks}$ evolutionary rates revealed that DUF506 genes are under purifying positive selection pressure. Subcellular protein localization analysis revealed that DUF506 proteins were present in different organelles. Transcript analyses showed that 13 of the Arabidopsis DUF506 genes are ubiquitously expressed in various tissues and respond to different abiotic stresses and ABA treatment. Protein-protein interaction network analysis using the STRING-DB, AtPIN (Arabidopsis thaliana Protein Interaction Network), and AI-1 (Arabidopsis Interactome-1) tools indicated that AtDUF506s potentially interact with iron-deficiency response proteins, salt-inducible transcription factors, or calcium sensors (calmodulins), implying that DUF506 genes have distinct biological functions including responses to environmental stimuli, nutrient-deficiencies, and participate in $\mathrm{Ca}(2+)$ signaling. Current results provide insightful information regarding the molecular features of the DUF506 family in plants, to support further functional characterizations.
\end{abstract}

Keywords: abiotic stress; arabidopsis; domain of unknown function (DUF); domain-structure; evolutionary analysis; expression profiling

\section{Introduction}

Domain of unknown function (DUF) is a conserved protein domain that has not been extensively characterized [1]. Designation as a DUF domain was derived from two bacterial motifs (DUF1 and 2), which were, respectively, renamed as GGDEF and EAL domains, based on their featured peptides [1,2]. The availability of whole genome sequence information has enabled the generation of a list of DUF-classified proteins in a wide range of organisms with a considerable portion (31\%, 5889 DUFs) found in the 19,179 entries in the Pfam database (34.0, https:/ / pfam.xfam.org/, accessed on August 2021) [3]. Numerous DUF genes have been studied in different species of plants. In Arabidopsis, DUF6 [4], DUF26 [5], DUF538 [6], DUF579 [7], DUF581 [8], DUF617/MIZ [9], DUF642 [10], DUF647 [11], DUF724 [12], DUF784 [13], DUF827 [14], DUF1117 [15] and DUF4228 [16] have been functionally characterized, and these studies have revealed the involvement of DUF-domain containing proteins in diverse biological processes. For example, At5g06700/TRICHOME BIREFRINGENCE/TBR and its homolog At5g01360/TRICHOME BIREFRINGENCE-LIKE 3/TBL3, which belong to the plant-specific DUF231 family, function in maintaining homeostasis of secondary wall cellulose synthesis in Arabidopsis [17]. Furthermore, another DUF231 member, At3g55990/ESK1/TBL29, was shown to be a negative regulator of freeze tolerance [18]. A recent evolutionary study revealed that DUF231 genes are found in all land plants and conduct conserved functions [19]. 
The DUF506 family belongs to the PD-(D/E)XK nuclease superfamily [20], which exists in all organisms. Most of the studies of DUF506 domain-containing proteins involve biochemical, mutagenesis, and structural biological approaches [21,22]. Currently, this superfamily includes approximately 22,900 proteins classified into 121 family branches [23]. However, due to low protein sequence similarity, DUF506 genes display extremely distant homologs among families [20] making it difficult to elucidate the molecular and biological functions of individual DUF506 genes. Recently, a phosphate-stress response DUF506 gene (At3g25240/REPRESSOR OF EXCESSIVE ROOT HAIR GROWTH 1/RXR1) in Arabidopsis was experimentally demonstrated to repress root hair elongation via interaction with RAB GTPase regardless of $P$ supplied status [24]. Furthermore, overexpression of the RXR1 homolog (Bradi2g58590) in the monocot model plant, Brachypodium, exhibited the identical short root hair phenotype, suggesting the conservative function of $R X R 1$ in regulation of plant cell development. Thus, the aim of this work was to conduct an extensive bioinformatics, expression profiling and subcellular analyses of plant DUF506 genes, to uncover their additional biological functions.

In this study, 211 DUF506 genes from 17 land plant species were identified with high confidence. These DUF506 genes were subjected to evolutionary analysis. In addition, subcellular localization, expression profiling analysis, and putative interactor(s) predictions of DUF506 family members not only extend the current knowledge of the PD-(D/E)XK superfamily in plants, but also provide beneficial insights for further studies into the distinct functions, such as abiotic stress responses, or regulation of calcium signaling.

\section{Results}

\subsection{Genome-Wide Survey and Phylogenetic Clustering of DUF506 Genes in Plants}

To extend current understanding of the DUF506 gene family, 323 putative DUF506 candidate gene sequences were extracted from the NCBI and Phytozome databases. Gene sequences encoding DUF506 domain-containing proteins were identified from 23 representative species, including Chlorophyta, Bryophyta, Lycophyta, Gymnospermae and Angiospermae (basal angiosperm, monocots and eudicots). Then, comprehensive protein sequences alignment [25] and domain alignment (HMM, Hidden Markov Model) [26] were respectively conducted to eliminate candidates with low integrity or incomplete domains. The remaining 266 DUF506 genes were then subjected to evolutionary analysis (Figure 1). Notably, no DUF506 genes were identified from Volvox carteri, and only one candidate was discovered from another chlorophyte (Chlamydomona reinhardtii). Generally, the dicot species have more DUF506 genes compared to monocots. The number of DUF506 gene sequences was highest in soybean (24), followed by two other legumes.

A phylogenetic tree was constructed to examine the evolutionary relationships among the 211 DUF506 candidates derived from 17 species in Angiosperms, using full-length protein sequence alignments. These DUF506 proteins were classified into three distinct groups (I,II, and III), consisting of 66, 51, and 94 members, respectively (Figure 2a, Table S1). Group III was further divided into two subgroups, named IIIla and IIIb, with 33 and 61 members, respectively. DUF506 proteins were found to be evenly distributed in each group (Table S2). For example, in Arabidopsis, there were four, two and seven DUF506 members in group I, group II, and group III, respectively. The lengths of Arabidopsis DUF506 protein ranged from 261 to 371 amino acid residues, while their predicted theoretical $\mathrm{pI}$ (Isoelectric point, the $\mathrm{pH}$ value at which the total charge on the protein is zero) varied from 5.02 to 8.96 (Table 1). Importantly, monocot and eudicot DUF506 proteins clustered into different branches (Figure 2a). These results indicate that classification of DUF506 members is largely concordant with the species tree (Figure 1).

\subsection{Motif Conservation within Diverse DUF506 Family Groups}

Within the DUF506 family, ten putative conserved motifs between five and one hundred amino acid residues were predicted using MEME [27]. The corresponding motif sequence logos and their distribution along the sequence are illustrated in Figure 2b,c. Gen- 
erally, group IIIa DUF506 members had the highest motif abundance. The most ubiquitous was motif 4 and was present in all (211/211) DUF506 proteins, while motif $1(204 / 211)$, motif $2(184 / 211)$, and motif $5(203 / 211)$ were present in most DUF506 proteins. In contrast, motifs 7 and 9 were exclusively found in group I and IIIa, respectively. Motifs 3, 6, 8, and 10 were present only in group IIIb. Overall, DUF506 members from the same groups generally share distinctive motif compositions, indicating that functional similarities within groups and the diversification among groups.

\subsection{Characterization of Chromosomal Distribution, Gene Synteny, and Sequence Structure of Arabidopsis DUF506 Genes}

The localization of DUF506 genes on Arabidopsis chromosomes was visualized through ePlant website (https: / / bar.utoronto.ca/ eplant/, accessed on May 2021). The thirteen Arabidopsis DUF506 genes were found on chromosomes 1-4 (Figure S1).

Gene synteny analysis revealed three segmental duplication events involving six Arabidopsis DUF506 genes (Figure S1, Table 2), whereas no tandem duplication was found. These results implied that the Arabidopsis DUF506 gene family expanded during evolution through gene segmental duplication. The ratio of nonsynonymous nucleotide substitutions (Ka), which led to amino acid altering, versus synonymous nucleotide substitutions (Ks), which did not change the amino acid sequence, was used to determine the selective pressure during genome evolution after gene duplication events [28]. Ka/Ks ratios for all three duplicated gene pairs are below 1, indicating that gene functional conservation during evolution, and suggesting that the Arabidopsis DUF506 gene family underwent purifying selection after the duplication events. Purifying selection of DUF506 genes was also found in monocot species, such as Brachypodium, rice, maize and sorghum (Table S4).

In addition, exon-intron distributions were examined to determine structural diversity among DUF506 genes by using GSDS 2.0. The results showed almost 58\% (122/211) of DUF506 genes contained more than two exons (Figure S2, and Table S3). For example, nine of the Arabidopsis DUF506 genes included three exons (Figure 3), and three duplicated gene pairs kept the identical exon-intron structure.

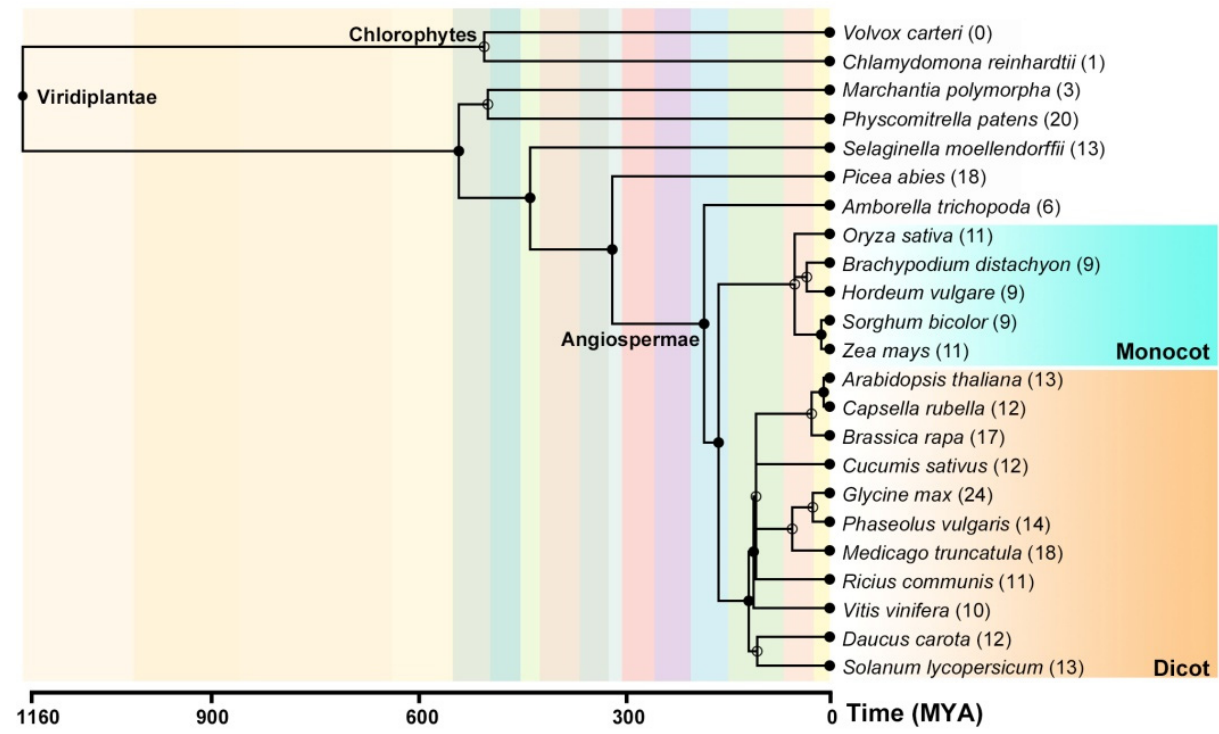

Figure 1. Phylogeny distribution of DUF506 genes across 23 species used in this study. The number of high confidence DUF506 genes is listed in parentheses. The order of tree branches and divergence time are derived from the TimeTree database (http:/ / www.timetree.org/, accessed on August 2021). Monocot species are highlighted in the rectangle of teal color, whereas the dicot species are masked by rectangle in yellow color. 
a

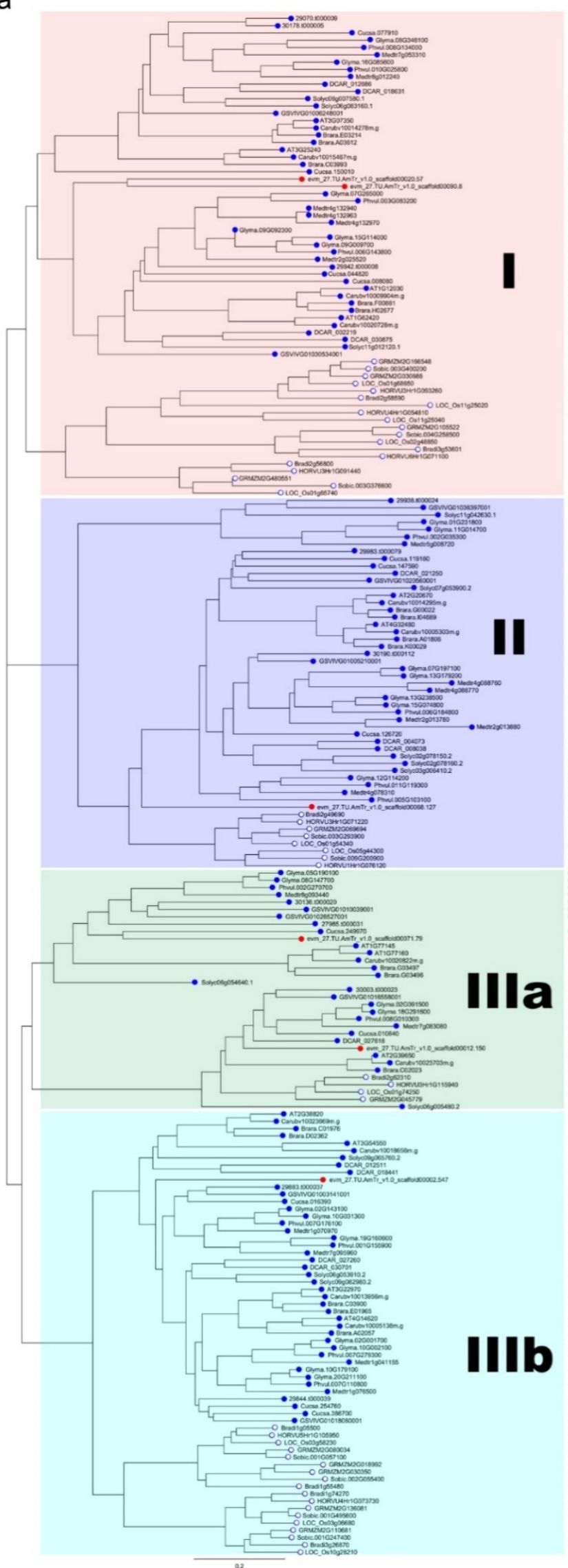

b
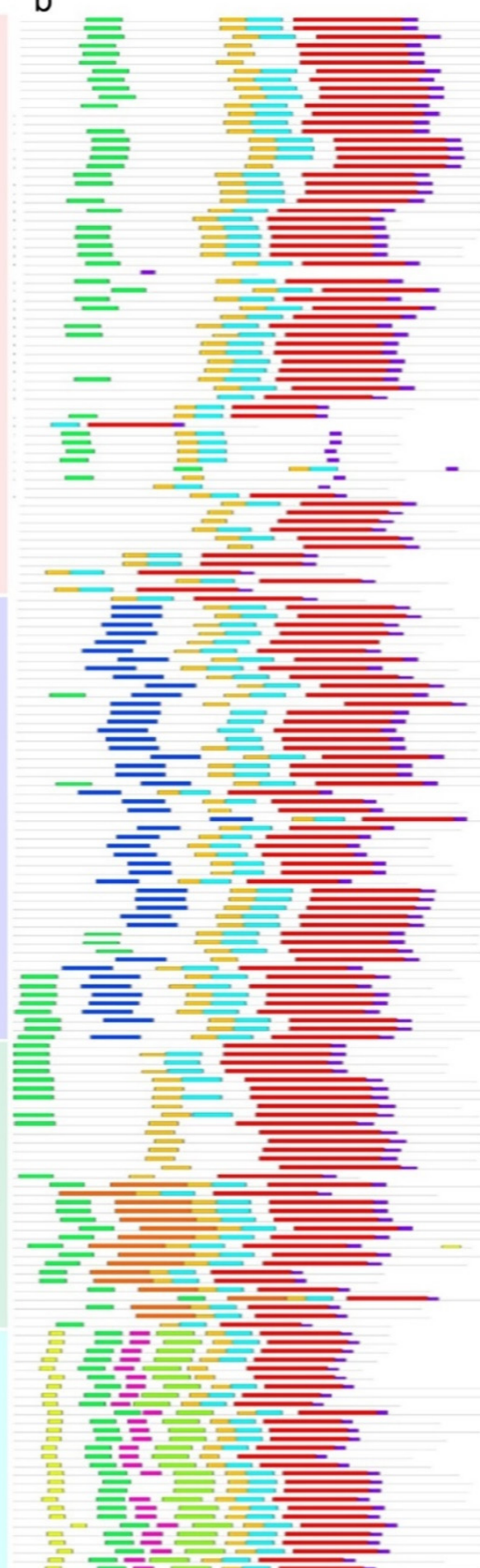

를 호를

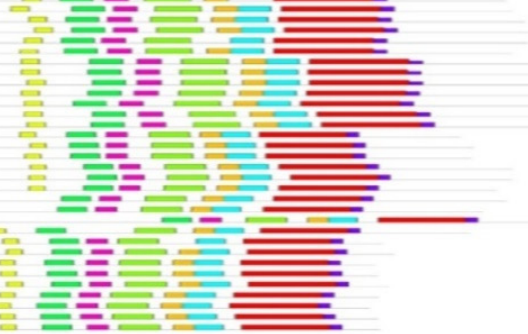




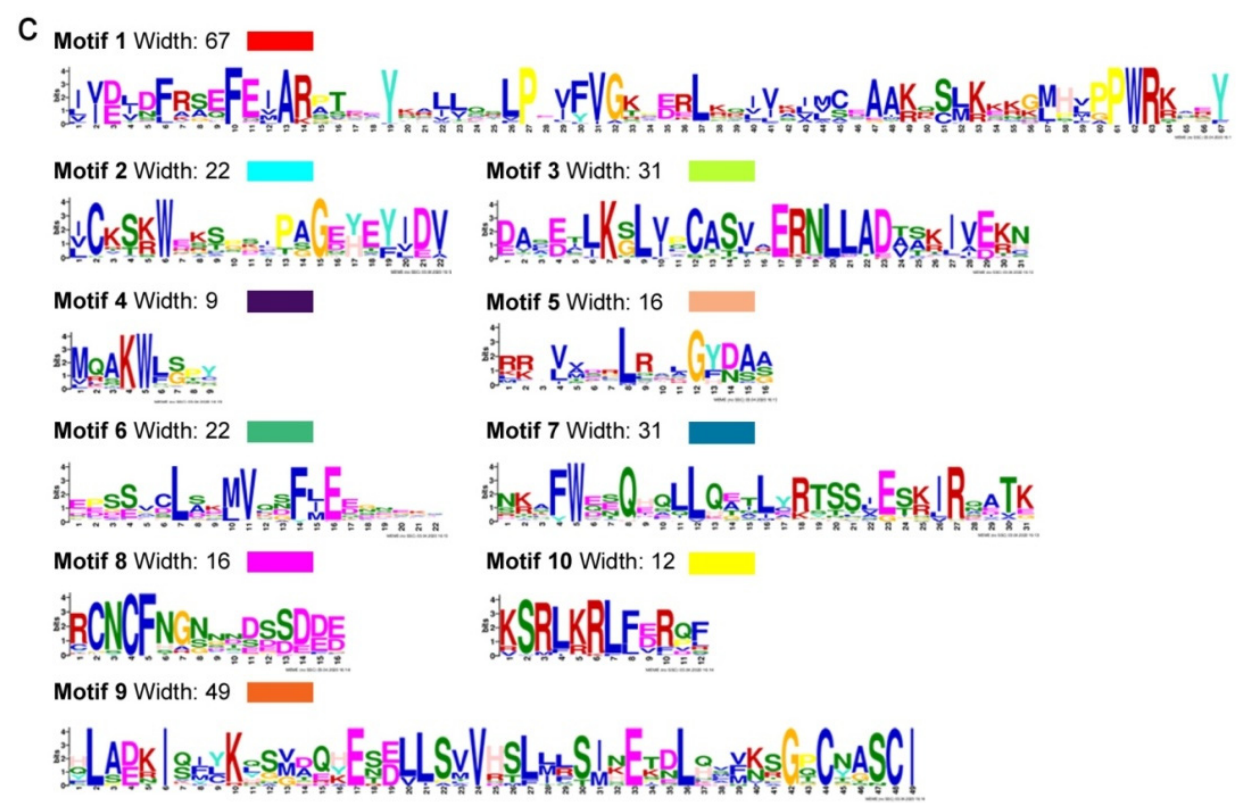

Figure 2. Phylogenetic representation and distribution of the conserved motifs in DUF506 among angiosperms. (a) The phylogenetic tree was constructed based on full-length DUF506 amino acid sequence alignments from 17 species. DUF506 from monocots are represented by blue hollow dots, while those from dicots are represented by blue solid dots. DUF506 from Amborella trichopoda (basal angiosperm) are represented by red solid dots. The distribution of DUF506 conserved motifs (b) and the corresponding sequence $\log$ os $(\mathbf{c})$ in plants are shown. Note that, the conserved $\beta$-sheet structures are mainly localized in Motif 2.

Table 1. Physicochemical properties of DUF506 family members in Arabidopsis.

\begin{tabular}{ccccl}
\hline GeneID & \# of A.A & p.I. & Subcellular Localization & Annotation (Phytozome v12.0) \\
\hline At1g12030 & 296 & 5.85 & cyto & phosphoenolpyruvate carboxylase \\
At1g62420 & 284 & 7.46 & chlo; cyto & DUF506 family protein \\
At1g77145 & 261 & 8.96 & nucl & transmembrane protein \\
At1g77160 & 264 & 8.26 & nucl & hypothetical protein \\
At2g20670 & 295 & 6.37 & nucl & sugar phosphate exchanger \\
At2g38820 & 311 & 8.53 & nucl & DNA-directed RNA polymerase subunit beta-beta protein \\
At2g39650 & 292 & 6.79 & nucl & cruciferin (DUF506) \\
At3g07350 & 299 & 5.47 & nucl & sulfate/thiosulfate import ATP-binding protein \\
At3g22970 & 371 & 5.02 & extr & hypothetical protein \\
At3g25240 & 282 & 7.61 & nucl & sulfate/thiosulfate import ATP-binding protein \\
At3g54550 & 289 & 8.63 & chlo & DNA-directed RNA polymerase subunit beta-beta protein \\
At4g14620 & 342 & 8.28 & cyto; cytoplas & hypothetical protein \\
At4g32480 & 288 & 7.66 & nucl & sugar phosphate exchanger \\
\hline
\end{tabular}

Table 2. Synteny analysis of DUF506 genes in Arabidopsis.

\begin{tabular}{cccccc}
\hline Duplicated Gene Pairs & Ka & Ks & Ka/Ks & Duplication Type & Type of Selection \\
\hline At1g12030/At1g62420 & 0.28 & 1.03 & 0.2718 & Segmental & Purify selection \\
At3g07350/At3g25240 & 0.46 & 1.76 & 0.2614 & Segmental & Purify selection \\
At3g22970/At4g14620 & 0.21 & 1.05 & 0.2000 & Segmental & Purify selection \\
\hline
\end{tabular}



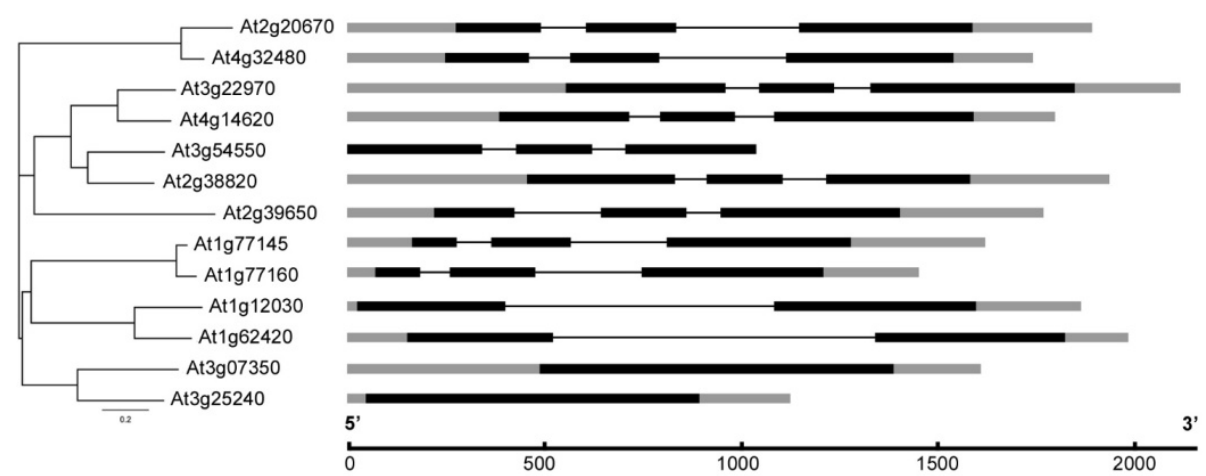

Figure 3. Phylogenetic analysis and exon-intron distribution of Arabidopsis DUF506 genes. Exon, intron and UTR are represented by black rectangle boxes, black lines and grey rectangle boxes, respectively.

\subsection{Wide Spread Localization of Arabidopsis DUF506 Proteins}

To study the subcellular localization of DUF506 proteins in Arabidopsis, six representative Arabidopsis DUF506 genes were cloned and transferred into the pEarleyGate 103 plasmid [29] to generate AtDUF506-GFP fused proteins driven by the CaMV35S promoter. Constructs were individually introduced into Arabidopsis mesophyll protoplasts using polyethylene glycol (PEG)-mediated transfection [30]. The resulting transient expression patterns are shown in Figure 4. The duplicated gene pairs were consistently expressed in identical organelles. For instance, At1g12030 and At1g62420 were localized in both the chloroplast and cytoplasm, while At3g22970 and At4g14620 were mainly in the plasma membrane, and slightly in the cytoplasm. Conversely, At3g25240 was concentrated in the nucleus and At3g07350 was present in the nucleus and cytoplasm. These results suggest that DUF506 proteins play diverse physiological roles based on their distinct patterns of organelle localization.

\subsection{Tissue Expression Profiles of Arabidopsis DUF506 Genes}

To characterize the expression patterns of the Arabidopsis DUF506 gene family, their transcript levels were examined in root, rosette leaves, stems, flowers, and siliques, using quantitative, real-time PCR (qRT-PCR). Arabidopsis DUF506 gene transcripts were detected in all tested tissues and exhibited different expression patterns (Figure 5). The At3g22970 transcript was abundant in all examined tissues, whereas the At3g25240 transcript had a comparably low abundance. A few DUF506 transcripts had much higher abundance in specific tissues or organs. For example, the At3g54550 transcript was at least 50 times more abundant in siliques than in flowers or roots. Interestingly, in adult plants the At1g62420 transcript was much more abundant in roots than in leaves, while in young seedlings, abundance was much higher in shoots than roots (Figure 6a). Such results suggest that DUF506 genes might perform different functions during development of Arabidopsis plants. 

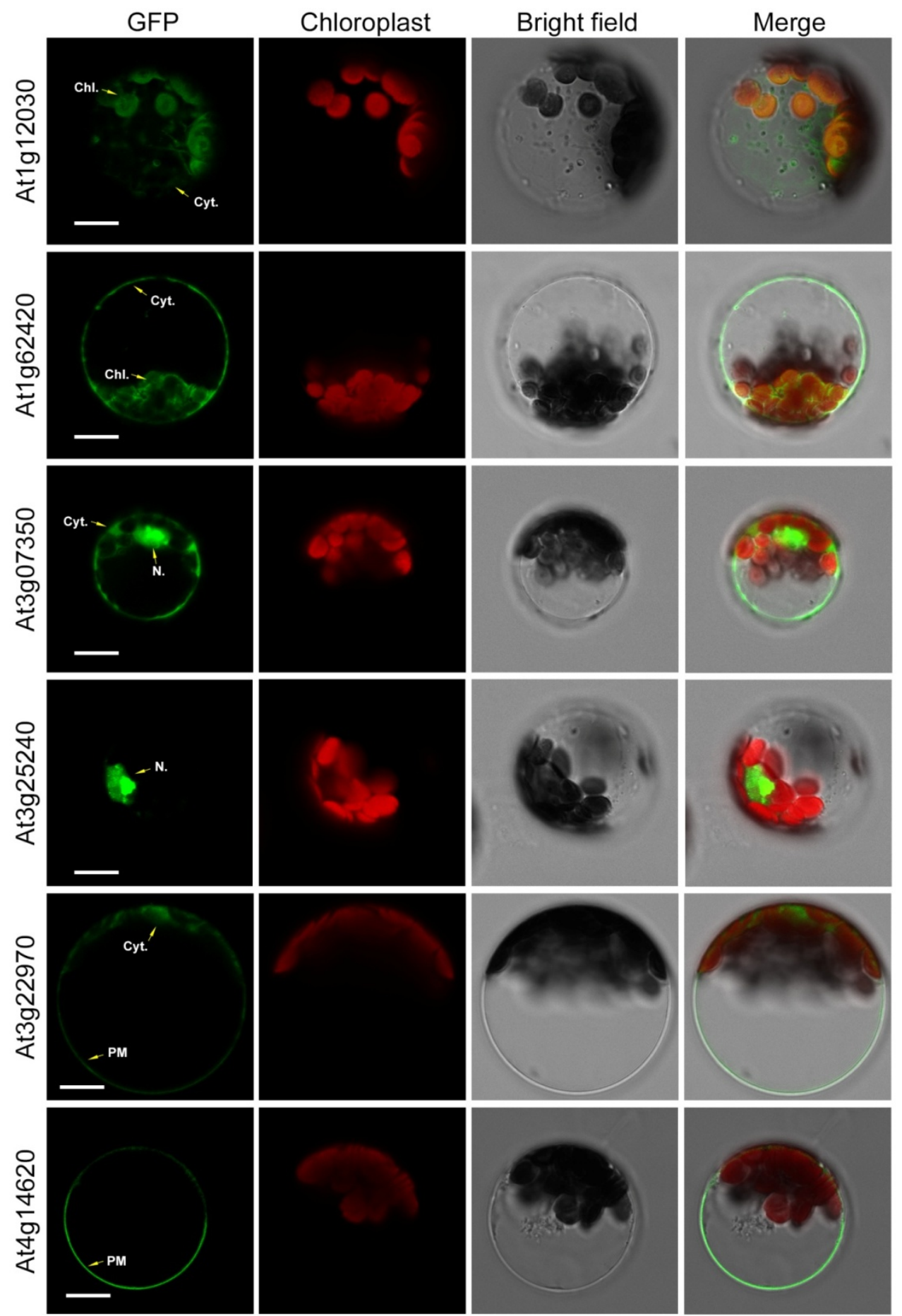

Figure 4. Subcellular localization of Arabidopsis DUF506 proteins visualized by transient expression of GFP fusion constructs in Arabidopsis mesophyll protoplasts. Chl., chloroplast; Cyt., cytoplasm; N., nucleus; PM, plasma membrane. Scale bar, $10 \mu \mathrm{m}$. 


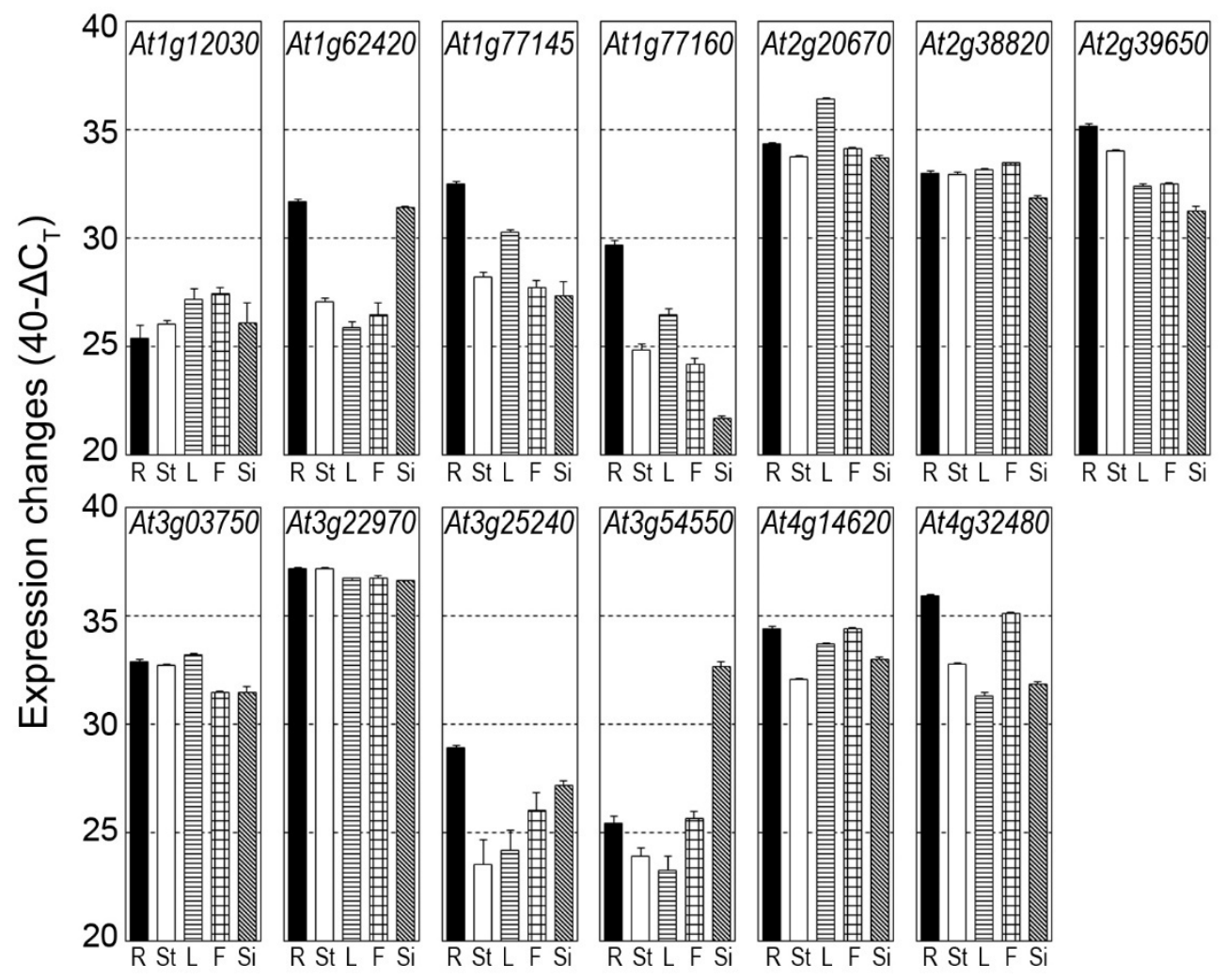

Figure 5. Expression profile of Arabidopsis DUF506 genes in different tissues. R, root; St, stem; L, leave; F, flower; Si, silique. Changes of gene expression level between tissues are interpreted as described in [31]. Expression levels are given on a log scale expressed as $40^{-\Delta C T}$, where $\Delta C T$ is the difference in qRT-PCR threshold cycle number between the respective gene and the reference gene (GAPDH); 40 therefore equals the expression level of GAPDH; the number 40 was chosen because the PCR run stops after 40 cycles. The fold difference in expression is $2^{\triangle \Delta C T}$ when PCR efficiency is 2 (e.g., an ordinate value of 34 represents 16 -fold lower expression than a value of 38 ).

\subsection{Response of Arabidopsis DUF506 Gene Transcripts to Abiotic Stress and Abscisic Acid Treatments}

Expression changes of Arabidopsis DUF506 genes in young seedlings, under salt, osmotic, and Abscisic acid (ABA) treatment were investigated (Figure 6a). Due to the low expression level in seedlings, the At3g54550 transcript was undetectable under all conditions. Under salt stress, At1g12030, At1g62420, At1g77160, At2g38820, At2g39650, and At3g25240 were highly induced in shoot and root tissues (fold change $[\mathrm{FC}] \geq 2$ ), whereas At1g77145 and At3g07350 were slightly up-regulated ( $1 \leq \mathrm{FC} \leq 2)$. By contrast, At2g20670, At3g22970 and At4g14620 were significantly down-regulated in shoots (FC $\geq 2$ ). Except for At1g62420 and At3g25240, no transcript changes were observed during osmotic stress.

Abscisic acid (ABA) has been shown to play an important role in stress responses and tolerance to abiotic stresses [32]. Most of the salt stress responsive Arabidopsis DUF506 genes, including At1g77145, At1g77160, At2g20670, At2g38820, At3g07350, and At4g14620, positively responded to ABA treatment, while At1g62420, At2g39650, and At3g25240 did not (Figure 6b). Meanwhile, expression of Arabidopsis DUF506 genes were explored under heat and cold stress available through online public transcriptome databases (GENEVESTIGATOR ${ }^{\circledR}$ and EMBL-EBI/ ArrayExpress). At3g25240 and At1g62420 were highly induced, but At2g20670 and At3g07350 were significantly repressed by both temperature stress conditions ( $\mathrm{FC} \geq 2$ ).

Stress-related cis-elements in the promoter regions ( $2 \mathrm{~kb}$ upstream of start codon) of the Arabidopsis DUF506 genes were also examined and identified (Figure S3). At least two copies of ABRE (ABA-responsive element) were found upstream of Arabidopsis DUF506 genes with the exception of At2g20670. The transcripts changes of the majority 
of Arabidopsis DUF506 genes (8/13, Figure 6b) under ABA treatment suggest that some of them might be involved in integrating ABA-related signaling pathways with abiotic stress responses.

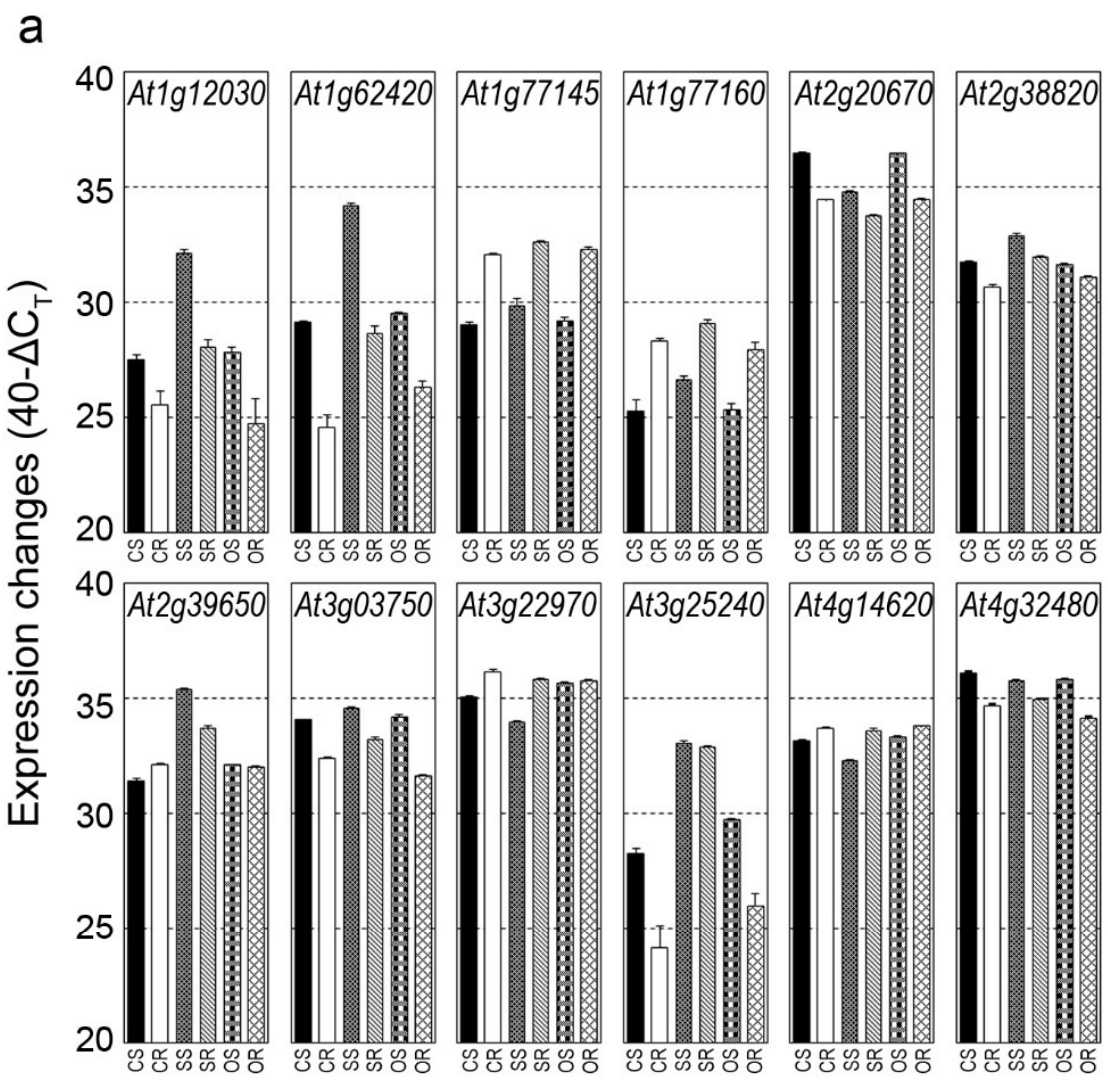

b

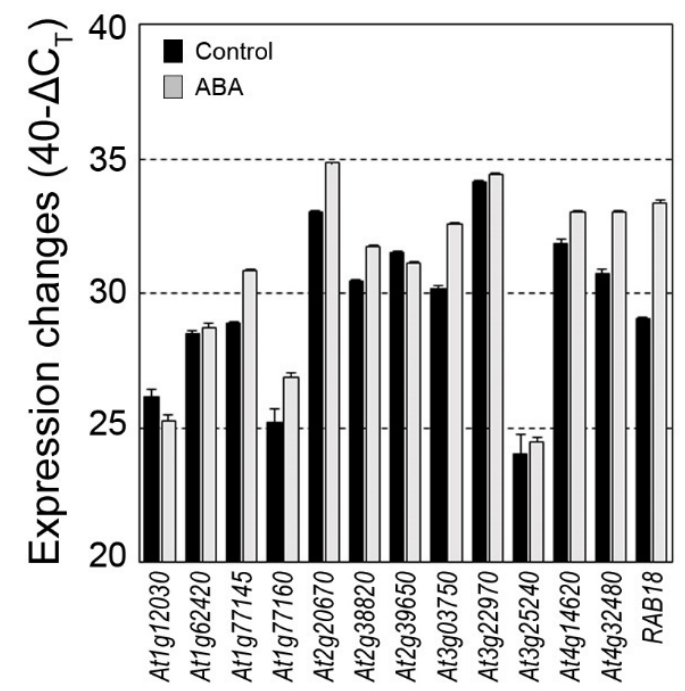

Figure 6. Expression profile of Arabidopsis DUF506 genes in response to salt or osmotic stress (a) and Abscisic acid (ABA) treatment (b). CS, control shoot tissue; CR, control root tissue; SS, salt stress shoot tissue; $\mathrm{SR}$, salt stress root tissue; OS, osmotic stress (mannitol) shoot tissue; OR, osmotic stress root tissue. At5g66400/RAB18, which is strongly induced by ABA, was used as positive external control (b). 


\subsection{Putative Interactors of Arabidopsis DUF506 Proteins}

To further uncover potential biological functions of DUF506 in Arabidopsis, proteinprotein interaction analysis was conducted using three different online search tools (Table S6). STRING-DB [33] (v11.0, https: / / string-db.org/, accessed on May 2021) identified seventyfive potential interactors for nine Arabidopsis DUF506 proteins, mainly based on coexpression profiles and text mining. Notably, several iron-deficiency related proteins (e.g., At1g47400/IRONMAM1, At5g67370/CGLD27, and At5g03570/IREG2) interacted with At1g12030, suggested its regulatory role in Fe signaling. Moreover, At2g39650 exhibited tight relationships with several salt stress regulators, such as salt-inducible ZINC Finger transcription factors (e.g., At3g55980/SZF1, At2g40140/SZF2, and At1g27730/ZAT10). On the other hand, the AtPIN and AI-1 programs only predicted two interactors of Arabidopsis DUF506 proteins. Remarkably, At1g77145 was predicted and has been experimentally proven to extensively interact with different calmodulins (CaMs, Figure 7, [34]), which are small calcium-binding proteins that function as second messengers in plant $\mathrm{Ca}^{2+}$ signaling [35]. The CaMs were identified from both databases, suggesting that At1g77145 might participate in $\mathrm{Ca}^{2+} / \mathrm{CaM}$ mediating signal transduction.

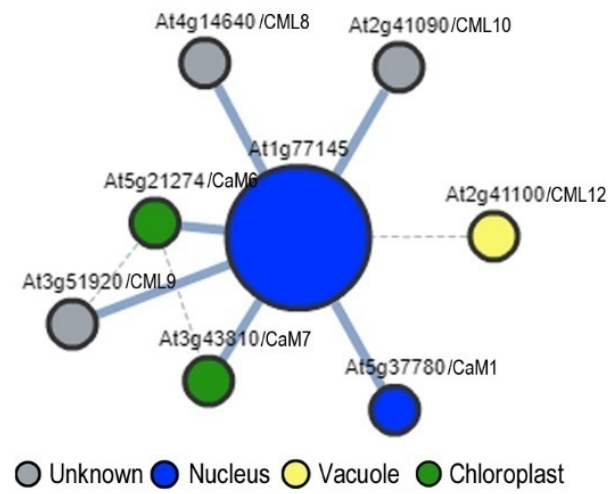

Figure 7. Predicted protein-protein interactions of At1g77145 and calmodulin/-like (CaM/CML). The interaction was predicted using AI-1 and the graph was generated by Arabidopsis interactions viewer (https: / / bar.utoronto.ca/interactions / cgi-bin/arabidopsis_interactions_viewer.cgi, accessed on June 2021). The color of nodes represents the subcellular localization predicted by SUBA (http: / / suba.live/, accessed on June 2021), and the thickness of lines represents the confident values of interactions. At2g41090, calmodulin like 10/CML10; At2g41100, calmodulin like 12/CML12; At3g43810, calmodulin 7/CaM7; At3g51920, calmodulin like 9/CML9; At4g14640, calmodulin like 8/CML8; At5g21274, calmodulin 6/CaM6; At5g37780, calmodulin 1/CaM1.

\section{Discussion}

Although the genome draft of Arabidopsis was released over two decades ago, more than 12,800 genes ( $46 \%$ of 27,655 protein-coding genes, Araport 11 , www.arabidopsis.org, accessed on March 2021) remain functionally uncharacterized. Intensive efforts have been devoted to identifying and characterizing these unknown or unannotated genes, such as DUFs. Several genes that contained various DUF domains have been studied in plants (e.g., TBL/DUF231). However, lack of genome-wide analysis of genes containing DUF domains has prevented a full understanding of their evolutionary history and biological functions.

\subsection{Evolution, Expansion and Conservation of Plant DUF506 Genes}

In the present study, examination of 23 representative species suggests that the green algae Chlamydomonas reinhardtii is the last common ancestor of DUF506 prior to plant colonization of the land (Figure 1). In bryophytes (e.g., M. polymorpha and P. patens), the number of DUF506 genes has largely increased, implying that they might have assisted with plant adaption to terrestrial conditions. Contrary to a recent report [16], the number of DUF506 genes in different species is closely coordinated with the total number of protein-coding transcripts, regardless of genome size (Table S5). Moreover, the species 
that belonged to the same taxa exhibited a similar percentage of DUF506 genes among the total genes (DUF506\%). For instance, between Arabidopsis and soybean, the difference of DUF506 (0.47\% versus $0.43 \%$ ) was significantly smaller when considering the genome size (135 Mb versus $978 \mathrm{Mb}$ ). Additionally, phylogenetic analysis found that in every subgroup, DUF506 proteins from monocots or eudicots tended to cluster in the same clades, suggesting the differentiation might be subjected before lineages diverged.

In the process of evolution, genome polyploidization has occurred frequently in Eukaryotes, concomitant with gene duplication events [36]. Three types of gene duplication events have been reported, namely tandem duplication, segmental duplication, and wholegenome duplication [37]. Here, three segmental duplicated gene pairs were identified from Arabidopsis, as well as in several monocot species (Table 2 and Table S4), suggesting that the expansion of the DUF506 gene family might have been derived from genome polyploidy events to diversify gene function. Noteworthy is that considering the chromosome positioning, phylogenetic relationship, identity of CDS and protein sequences, At1g77145 and $A t 1 g 77160$ could be an undiscovered tandem duplicated gene pair (Figures 1, 2a and 3; Table 1). Previous studies have demonstrated essential and multifaceted genes, such as transcription factors and protein kinases, were retained during natural selection, whereas genes with a single specialized biological function, such as transferases, had been preferentially lost [38]. At3g25240/RXR1, and its duplicated pair, At3g07350, both strongly responded to P-stress [24]. However, they showed different expression patterns when exposed to abiotic stresses or ABA treatment (Figure 6). Taken together, the present results show that DUF506 genes from same species have experienced strong purifying positive selection after the duplication event $(\mathrm{Ka} / \mathrm{Ks}$ ratio $<1$, Table 2$)$, implicating their functional redundancy under certain conditions.

Structural-modeling (secondary and tertiary structures) is increasingly considered more effective at predicting protein function than protein sequencing (primary structure) alone, because the conservation of functional foldable motifs is likely higher than the sequence $[8,39]$. A previous study predicted DUF506 proteins contain a conserved $(\alpha \alpha \alpha \alpha \beta \beta \beta \alpha \beta \alpha \alpha)$ secondary structure pattern [20]. Comparative structural analysis found the three tandem $\beta$-sheet structures were mainly presented in motif 2 (Figure $2 \mathrm{c}$ ), which is found in most DUF506 proteins, whereas the $\alpha$-coil structures were scattered in the neighborhood. Considering the ubiquitous presence of these secondary structures, DUF506s might perform conserved biological functions across species.

\subsection{Distinct Roles of DUF506 Genes in Biological Processes}

Expression profiling of Arabidopsis DUF506 genes in different tissues, developmental stages, and under abiotic stress provided new data that could help in understanding potential biological functions. In general, transcript levels of DUF506 genes fluctuated in various organs (Figures 5 and 6a). The hypothetical tandem duplicated gene pair, At1g77145 and $A t 1 g 77160$, displayed identical expression patterns. These results supported the hypothesis of positive selection of DUF506 genes based on the evolutionary analysis. However, expression changes of the segmental duplicated pair diverged from that of its homolog. For example, the At1g62420 transcript was highly abundant in roots and siliques relative to other tissues, whereas the At1g12030 transcript was steadily expressed.

The Arabidopsis DUF506 transcripts responded more to salt stress than to osmotic stress. As shown in Figure 6a, most Arabidopsis DUF506 genes (10/12, except At3g54550) responded to salt stress, whereas only two genes (At1g62420 and $A t 3 g 25240)$ were induced by osmotic treatment. Additionally, about $75 \%(8 / 12)$ of the genes responded to ABA treatment, an observation that was consistent with the presence of ABRE cis-elements in their promoters (Figure S3). At1g12030, At1g77160, At2g20670, and At2g38820, significantly responded to both salinity and ABA treatment. At1g62420 and At3g25240 transcripts were strongly induced by distinct abiotic stresses (e.g., salt, osmotic, cold, and heat), but were irresponsive to ABA treatment despite the presence of ABREs in their promoter regions (Figure 5, Figure 6 and Figure S3). Overall, these results suggest that the expression of 
Arabidopsis DUF506 genes are mediated through both ABA-dependent (e.g., At1g12030 and At1g77160) and independent (e.g., At1g62420 and At3g25240) pathways in response to environmental stimuli.

DUF506 genes in other plant species actively responded to abiotic stress (Figures S4-S9). For example, in Brachypodium and rice, Bradi2g58590 and LOC_Os01g68650, the closest homologs of At3g25240, were strongly up-regulated by salinity and cold stress. A similar expression pattern was observed in At2g39650 homologs (Bradi2g62310 and LOC_Os01g74250). Due to limited data availability on soybean and Medicago, the expression profiles of DUF506 genes responding to drought stress were analyzed. Medtr1g070970, Medtr2g013780, Medtr4g088770, Medtr8g093440, and their corresponding homologs in Soybean, Glyma.02G143100, Glyma.15G074800, Glyma.13G179200, Glyma.05G190100 were significantly induced by drought stress.

Nowadays, protein interaction analysis, through yeast two hybridization $(\mathrm{Y} 2 \mathrm{H})$ or prediction based on public inter-proteomic data, is a useful tool to provide additional insights deciphering gene functions. At1g12030, which is the only DUF506 gene that clearly responds to iron starvation (Fold Change $[F C] \geq 6$ in shoot, and FC $\geq 2.5$ in root, GENEVESTIGATOR ${ }^{\circledR}$ ), was predicted to interact with several iron (Fe)-deficiency response proteins (Table S6). One example is At1g47400/FEP3/IRONMAN1, which encodes a 50 amino acid long FE-UPTAKE-INDUCING PEPTIDE, predominantly expressed in the vascular tissue (e.g., phloem) of leaves and roots. Overexpression of FEP3 significantly accumulated Fe and $\mathrm{Mn}$ in Arabidopsis through activation of Fe uptake genes in roots [40,41]. Additional Fe-deficiency related proteins that interactin with At1g12030, including chloroplast-localized At5g67370/CONSERVED IN THE GREEN LINEAGE AND DIATOMS 27/CGLD27, 14-3-3 protein At1g34760/ROOT HAIR SPECIFIC 5/RHS5, tonoplast-localized iron efflux transporter At5g03570/IRON-REGULATED PROTEIN 2/IREG2, and phloem-based iron transporter At1g56430/NICOTIANAMINE SYNTHASE 4/NAS4, have been demonstrated to function effectively in maintaining endogenous iron homeostasis [42-45]. Lately, a novel basic helix-loop-helix (bHLH) transcription factor, At3g19860/Upstream Regulator of IRT1/URI, was demonstrated to act as a central regulator of the iron deficient signaling pathway in Arabidopsis [46]. Chromatin immunoprecipitation followed by a sequencing (ChIP-seq) experiment revealed that URI directly bound to promoters of many iron-regulated genes, including At1g12030 and its interactors, At5g05250/IRP6, IMA1, CGLD27, and NAS4 [46]. These results suggest that At1g12030 acts in an essential URI-dependent role in responding to iron deficiency.

In addition to responding nutrient-deficiency, another Arabidopsis DUF506 member, At2g39650, intensively interacted with four well-known salt-stress-related transcription factors, including SZF1, SZF2, ZAT10, and At3g49530/NAC062 (Table S6). Three of the ZINC finger domain-containing proteins have been thoroughly characterized and were proven to be regulators under multiple stresses (e.g., salt, drought, cold, hypoxia, and $\mathrm{H}_{2} \mathrm{O}_{2}$ ) by activating the expression of stress-inducible genes [47-49]. Moreover, the membrane-bound transcription factor NAC062 was reported not only to promptly activate transcriptional responses upon exposure to abiotic/biotic stress, but also to mediate the unfolded protein response (UPR) under endoplasmic reticulum (ER) stress [50,51]. Thus, considering the high induction of At2g39650 under salt stress (Figure 6a), the present results suggest its involvement in transcriptional regulation of stress-response genes by interacting with different transcription factors.

Unlike the aforementioned interactions predicted based on text mining and co-expression data, At1g77145's interaction with at least five Calmodulin/-like proteins (CaM/CML), such as CML8, CML9, CML10, CaM6, and CaM7, was determined through protein microarrays [34]. In plants, when intracellular levels of $\mathrm{Ca}^{2+}$ increase, CaM/CML directly binds to $\mathrm{Ca}^{2+}$ via symmetrical EF-hand motifs, then functions as an essential calcium secondary messenger by altering the activity of target proteins to contribute to signaling during developmental processes and adaptation to environmental stimuli [52]. CML8, CaM6, and CaM7 have been demonstrated to interact with the BRASSINOSTEROID-INSENSITIVE 1 
(BRI1) receptor kinase [53]. The interactions inhibited the activity of BRI1, suggesting the participation of $\mathrm{Ca}^{2+}$ in BR signaling. Phylogenic analysis indicated that CML8, CML9, and CML10 belong to the same CML subgroup in Arabidopsis, and actively responded to various environmental stimuli [54]. The CML8 transcript has been reported to be induced by salicylic acid (SA) and salt treatment [55]. CML9 mutants exhibited hypersensitivity to ABA, and elevated salt and drought tolerance through up-regulation of stress-response genes, suggesting that AtCML9 is involved in the ABA-dependent pathway responding to abiotic stress [56]. Differently, through interacting with the Ascorbic acid (AsA) biosynthesis-related enzyme phosphomannomutase (PMM), CML10 augmented the activity of PMM and consequently modulated oxidative stress responses [57]. Moreover, CML8 and CML9 have been reported to play roles in plant immune response [58,59]. Searching the Calmodulin Target Database [60], a classic 1-12 motif ([FILVW]xxxxxxxxxx[FILVW], two bulky hydrophobic residues spaced by 10 amino acids residues) was identified in motif 1 of At1g77145. Further analysis showed different types of CaM binding motifs were scattered in twelve of the Arabidopsis DUF506 proteins (Table S7). Presumably, At1g77145, and perhaps other DUF506 members, might specifically bind to certain CaMs/CMLs in the presence of $\mathrm{Ca}^{2+}$, leading to a structural conformation change or a shift in enzymatic activity, and ultimately influencing downstream stress-response gene expression.

The silique-specific At3g54550 potentially interacts with At2g38820 (Table S6), which is constitutively expressed in vivo, implying a possible chimeric complex consisting of different DUF506 proteins, might perform regulatory functions during the reproductive stage, especially during seed development.

Overall, the broad response of DUF506 genes to various abiotic stresses and the predicted interaction with various functional proteins suggest that Arabidopsis DUF506 proteins have a diverse range of regulatory functions in response to abiotic stress (e.g., At2g39650 and At1g77145) or nutrient deficiency (e.g., At1g12030 and At3g25240).

\section{Materials and Methods}

\subsection{Identification and Phylogenetic Analysis of DUF506 Protein in Plants}

In this study, 211 DUF506 candidate genes (Pfam accession: PF04720, ID: PDDEXK_6, Description: PDDEXK-like family of unknown function, https://pfam.xfam.org/) were identified from 17 plant species. The protein sequences, cDNA sequences, DNA sequences, upstream $2 \mathrm{~kb}$ genomic DNA sequences, and protein-coding sequences (CDS) of DUF506 were downloaded from Phytozome v12.1.6 (Amborella trichopoda v1.0, Arabidopsis thaliana Araport11, Capsella rubella v1.0, Brassica rapa FPsc v1.3, Cucumis satious v1.0, Glycine max Wm82.a2.v1, Phaseolus vulgaris v2.1, Medicago truncatula Mt4.0 v1, Ricinus communis v0.1, Vitis vinifera Genoscope.12X, Daucus carota v2.0, Solanum lycopersicum iTAG2.4, Oryza sativa v7_JGI, Brachypodium distachyon v3.1, Hordeum vulgare r1, Sorghum bicolor v3.1.1, and Zea mays PH207 v1.1). In genes undergoing alternative splicing, the longest transcript was used for downstream analysis. Predicted partial proteins carrying the signature domain were filtered out. The theoretical isoelectric point (pI) of DUF506 proteins were calculated by Sequence Manipulation Suite (https: / / www.bioinformatics.org/sms2/, Sequence analysis tool-Protein Isoelectric Point) [61]. The subcellular localization was predicted by using WoLF PSORT program (https:/ / wolfpsort.hgc.jp/).

The amino acid sequence alignment of DUF506 genes was performed by Clustal Omega1.2.2 (https:/ / www.ebi.ac.uk/Tools/msa/clustalo/). The alignment was used to construct an unrooted phylogenic tree using the neighbor-joining method with 1000 bootstrap replications using Fast Tree. Both programs were carried out within Geneious Prime ${ }^{\circledR}$ software (v.2020.2.3.).

\subsection{Gene Structure and Conserved Motif Analysis}

The exon-intron distributions of DUF506 genes were graphically generated by Gene Structure Display Server 2.0 [62] (http:/ / gsds.gao-lab.org/) using coding sequences and their corresponding genomic DNA sequences under default settings. The conserved 
motifs of DUF506 protein were predicted by using the MEME web server [27] (v5.1.1, http:/ / meme-suite.org/tools/meme) with the maximum number of motifs set at 10, and optimum width of motifs between 5 and 100 amino acids. The secondary structure of DUF506 protein was predicted by using the JPred4 web server [63] (A Protein Secondary Structure Prediction Server, http:/ / www.compbio.dundee.ac.uk/jpred4/index_up.html).

\subsection{Chromosomal Localization, Gene Duplication and Syntenic Analysis}

Chromosomal locations of Arabidopsis DUF506 proteins were visualized by using ePlant web tool (https:/ / bar.utoronto.ca/eplant/). Gene duplication events were analyzed by using the Multiple Collinearity Scan toolkit [64]. The precalculated nonsynonymous (Ka) and synonymous (Ks) substitutions of each duplicated Arabidopsis DUF506 genes were obtained from Plant Genome Duplication Database (PGDD, http:/ / chibba.agtec.uga. edu/duplication/).

\subsection{Subcellular Localization of Arabidopsis DUF506 Protein}

The coding regions, except for the terminator codon of selected Arabidopsis DUF506 genes, were amplified and then ligated into the destination vector, pEarleyGate 103 [29], to generate a AtDUF506-GFP fusion construct under the control of the CaMV35S promoter. Constructs were transformed into Arabidopsis (Col-0) mesophyll protoplast as described previously [30]. Twelve hours after transformation, fluorescence was detected using a Leica TCS SP8 confocal laser-scanning microscope. All experiments were repeated at least three times and representative images are displayed in figures.

\subsection{Stress-Related Cis-Elements Analysis}

The PlantCARE program [65] (http:/ / bioinformatics.psb.ugent.be/webtools/plantcare/ $\mathrm{html} /$ ) was employed to analyze the $2 \mathrm{~kb}$ upstream genomic sequences of plant DUF506 genes.

\subsection{Expression Analysis of Arabidopsis DUF506 Genes via qRT-PCR}

Arabidopsis thaliana (ecotype Col-0) seeds were sterilized in $70 \%$ ethanol and 20\% bleach as described previously [66]. After stratification for 3 days at $4{ }^{\circ} \mathrm{C}$, sterilized seeds were directly sown in half strength (1/2) Murashige and Skoog (MS) medium (Caisson Labs) supplemented with $1 \%$ sucrose ( $\mathrm{pH} 5.7)$ and $0.05 \% \mathrm{MES}(w / v)$. Seeds were germinated and grown in a growth chamber maintained at $22^{\circ} \mathrm{C}$ (16-h-light and 8-h-dark cycle, $120 \mu \mathrm{mol}^{-2} \mathrm{~s}^{-1}$ light intensity). The roots and shoots of 10 -day-old seedlings, and the roots, stems, rosette leaves, flowers and siliques of mature Arabidopsis plants were used for tissue-specific expression analysis. For salt stress, osmotic stress, or ABA treatment, 7-day-old Arabidopsis seedling were transferred into 1/2 MS liquid medium supplemented with $150 \mathrm{mM} \mathrm{NaCl}, 300 \mathrm{mM}$ mannitol or $100 \mu \mathrm{M}( \pm)$-cis, trans-ABA (Sigma), and the stress treatments were applied for $24 \mathrm{hr}$. A fresh medium-only control was conducted in parallel. Samples were rinsed with demineralized water, shoot and root tissues were harvested separately, snap-frozen in liquid $\mathrm{N}_{2}$ and stored at $-80{ }^{\circ} \mathrm{C}$ until further use.

The qRT-PCR experiments were performed according to previous studies [66]. All the experiments were repeated at least three times using cDNA prepared from two biological replicates. Primers used in the study are listed in Supplemental Table S8.

\subsection{Protein-Protein Interaction Analysis}

STRING-DB [33] (v11.0, https:/ / string-db.org/), AtPIN [67] (Arabidopsis thaliana protein interaction network, https:/ / atpin.bioinfoguy.net/cgi-bin/atpin.pl), and AI-1 [68] (Arabidopsis Interactome-1, http:/ /interactome.dfci.harvard.edu/A_thaliana/index.php) online search tools were used to predict the putative protein-protein interaction networks with candidate Arabidopsis DUF506 proteins using default settings. 


\section{Conclusions}

In this study, 211 DUF506 genes in 17 land species were identified and were conducted to estimate evolutionary analysis. Gene structure and conserve motif analysis indicated the conservation and dispersal of DUF506 during evolution. Furthermore, expression profiling and protein interaction predictions suggest that Arabidopsis DUF506 genes might be involved in plant resilience to environmental stresses.

Supplementary Materials: The following are available online at https:/ /www.mdpi.com/article/10 $.3390 /$ ijms222111442/s1.

Funding: The work was funded by the Noble Research Institute LLC.

Institutional Review Board Statement: Not applicable.

Informed Consent Statement: Not applicable.

Data Availability Statement: The data and materials that support the findings of this study are available from the corresponding author upon reasonable request.

Acknowledgments: The author thanks Sylvia Warner (Noble Research Institute) for lab assistance, Wolf Scheible (Noble Research Institute) and Elison Blancaflor (NASA) for critical comments of the manuscript.

Conflicts of Interest: The authors declare no conflict of interest.

\section{References}

1. Bateman, A.; Coggill, P.; Finn, R. DUFs: Families in search of function. Acta Crystallogr. Sect. F Struct. Biol. Cryst. Commun. 2010, 66, 1148-1152. [CrossRef]

2. Simm, R.; Morr, M.; Kader, A.; Nimtz, M.; Römling, U. GGDEF and EAL domains inversely regulate cyclic di-GMP levels and transition from sessility to motility. Mol. Microbiol. 2004, 53, 1123-1134. [CrossRef]

3. Mistry, J.; Chuguransky, S.; Williams, L.; Qureshi, M.; Salazar, G.A.; Sonnhammer, E.L.L.; Tosatto, S.C.E.; Paladin, L.; Raj, S.; Richardson, L.J.; et al. Pfam: The protein families database in 2021. Nucleic Acids Res. 2020, 49, D412-D419. [CrossRef] [PubMed]

4. Ranocha, P.; Denancé, N.; Vanholme, R.; Freydier, A.; Martinez, Y.; Hoffmann, L.; Köhler, L.; Pouzet, C.; Renou, J.-P.; Sundberg, B.; et al. Walls are thin 1 (WAT1), an Arabidopsis homolog of Medicago truncatula NODULIN21, is a tonoplast-localized protein required for secondary wall formation in fibers. Plant J. 2010, 63, 469-483. [CrossRef] [PubMed]

5. Vaattovaara, A.; Brandt, B.; Rajaraman, S.; Safronov, O.; Veidenberg, A.; Luklová, M.; Kangasjärvi, J.; Löytynoja, A.; Ho-thorn, M.; Salojärvi, J.; et al. Mechanistic insights into the evolution of DUF26-containing proteins in land plants. Commun. Biol. $2019,2,56$. [CrossRef]

6. Yu, C.-Y.; Sharma, O.; Nguyen, P.H.T.; Hartono, C.D.; Kanehara, K. A pair of DUF538 domain-containing proteins modulates plant growth and trichome development through the transcriptional regulation of GLABRA1 in Arabidopsis thaliana. Plant J. 2021. Accepted Author Manuscript. [CrossRef] [PubMed]

7. Urbanowicz, B.R.; Peña, M.J.; Ratnaparkhe, S.; Avci, U.; Backe, J.; Steet, H.F.; Foston, M.; Li, H.; O’Neill, M.A.; Ragauskas, A.J.; et al. 4-O-methylation of glucuronic acid in Arabidopsis glucuronoxylan is catalyzed by a domain of unknown function family 579 protein. Proc. Natl. Acad. Sci. USA 2012, 109, 14253-14258. [CrossRef] [PubMed]

8. Laxmi, A. DUF581 Is Plant Specific FCS-Like Zinc Finger Involved in Protein-Protein Interaction. PLoS ONE 2014, 9, e99074. [CrossRef]

9. Moriwaki, T.; Miyazawa, Y.; Kobayashi, A.; Uchida, M.; Watanabe, C.; Fujii, N.; Takahashi, H. Hormonal Regulation of Lateral Root Development in Arabidopsis Modulated by MIZ1 and Requirement of GNOM Activity for MIZ1 Function. Plant Physiol. 2011, 157, 1209-1220. [CrossRef] [PubMed]

10. Zúñiga-Sánchez, E.; Soriano, D.; Martínez-Barajas, E.; Orozco-Segovia, A.; Gamboa-deBuen, A. BIIDXI, the At4g32460 DUF642 gene, is involved in pectin methyl esterase regulation during Arabidopsis thaliana seed germination and plant development. BMC Plant Biol. 2014, 14, 338. [CrossRef]

11. Tong, H.; Leasure, C.D.; Hou, X.; Yuen, G.; Briggs, W.; He, Z.-H. Role of root UV-B sensing in Arabidopsis early seedling development. Proc. Natl. Acad. Sci. USA 2008, 105, 21039-21044. [CrossRef] [PubMed]

12. Cao, X.; Yang, K.-Z.; Xia, C.; Zhang, X.-Q.; Chen, L.-Q.; Ye, D. Characterization of DUF724 gene family in Arabidopsis thaliana. Plant Mol. Biol. 2009, 72, 61. [CrossRef]

13. Jones-Rhoades, M.W.; Borevitz, J.O.; Preuss, D. Genome-wide expression profiling of the Arabidopsis female gametophyte identifies families of small, secreted proteins. PLoS Genet. 2007, 3, 1848-1861. [CrossRef]

14. Kodama, Y.; Suetsugu, N.; Wada, M. Novel protein-protein interaction family proteins involved in chloroplast movement response. Plant Signal. Behav. 2011, 6, 483-490. [CrossRef] 
15. Kim, S.J.; Ryu, M.Y.; Kim, W.T. Suppression of Arabidopsis RING-DUF1117 E3 ubiquitin ligases, AtRDUF1 and AtRDUF2, reduces tolerance to ABA-mediated drought stress. Biochem. Biophys. Res. Commun. 2012, 420, 141-147. [CrossRef]

16. Yang, Q.; Niu, X.; Tian, X.; Zhang, X.; Cong, J.; Wang, R.; Zhang, G.; Li, G. Comprehensive genomic analysis of the DUF4228 gene family in land plants and expression profiling of ATDUF4228 under abiotic stresses. BMC Genom. 2020, 21, 12-16. [CrossRef]

17. Bischoff, V.; Nita, S.; Neumetzler, L.; Schindelasch, D.; Urbain, A.; Eshed, R.; Persson, S.; Delmer, D.; Scheible, W.-R. TRI-CHOME BIREFRINGENCE and Its Homolog AT5G01360 Encode Plant-Specific DUF231 Proteins Required for Cellulose Bi-osynthesis in Arabidopsis. Plant Physiol. 2010, 153, 590-602. [CrossRef] [PubMed]

18. Xin, Z.; Mandaokar, A.; Chen, J.; Last, R.L.; Browse, J. Arabidopsis ESK1 encodes a novel regulator of freezing tolerance. Plant J. 2007, 49, 786-799. [CrossRef]

19. Zhong, R.; Cui, D.; Ye, Z. Evolutionary origin of $O$-acetyltransferases responsible for glucomannan acetylation in land plants. New Phytol. 2019, 224, 466-479. [CrossRef] [PubMed]

20. Knizewski, L.; Kinch, L.N.; Grishin, N.V.; Rychlewski, L.; Ginalski, K. Realm of PD-(D/E)XK nuclease superfamily revisited: Detection of novel families with modified transitive meta profile searches. BMC Struct. Biol. 2007, 7, 40. [CrossRef] [PubMed]

21. Kosinski, J.; Feder, M.; Bujnicki, J.M. The PD-(D/E)XK superfamily revisited: Identification of new members among proteins involved in DNA metabolism and functional predictions for domains of (hitherto) unknown function. BMC Bioinform. 2005, 6, 172. [CrossRef]

22. Zhao, L.; Bonocora, R.P.; Shub, D.A.; Stoddard, B.L. The restriction fold turns to the dark side: A bacterial homing endonu-clease with a PD-(D/E)-XK motif. EMBO J. 2007, 26, 2432-2442. [CrossRef]

23. Steczkiewicz, K.; Muszewska, A.; Knizewski, L.; Rychlewski, L.; Ginalski, K. Sequence, structure and functional diversity of PD-(D/E)XK phosphodiesterase superfamily. Nucleic Acids Res. 2012, 40, 7016-7045. [CrossRef]

24. Ying, S.; Blancaflor, E.; Liao, F.; Scheible, W.R. A Phosphorus-Limitation Induced, Functionally Conserved DUF506 Protein is a Repressor of Root Hair Elongation in Arabidopsis thaliana. bioRxiv 2021. [CrossRef]

25. Edgar, R.C. MUSCLE: Multiple sequence alignment with high accuracy and high throughput. Nucleic Acids Res. 2004, 32, 1792-1797. [CrossRef] [PubMed]

26. Mount, D.W. Using Hidden Markov Models to Align Multiple Sequences. Cold Spring Harb. Protoc. 2009, 2009 , pdb.top41. [CrossRef] [PubMed]

27. Bailey, T.L.; Boden, M.; Buske, F.A.; Frith, M.; Grant, C.E.; Clementi, L.; Ren, J.; Li, W.W.; Noble, W.S. MEME SUITE: Tools for motif discovery and searching. Nucleic Acids Res. 2009, 37, w202-w208. [CrossRef]

28. Fay, J.C.; Wu, C.-I. Sequence Divergence, Functional Constraint, and Selection in Protein Evolution. Annu. Rev. Genom. Hum. Genet. 2003, 4, 213-235. [CrossRef] [PubMed]

29. Earley, K.W.; Haag, J.R.; Pontes, O.; Opper, K.; Juehne, T.; Song, K.; Pikaard, C.S. Gateway-compatible vectors for plant functional genomics and proteomics. Plant J. 2006, 45, 616-629. [CrossRef] [PubMed]

30. Yoo, S.-D.; Cho, Y.-H.; Sheen, J. Arabidopsis mesophyll protoplasts: A versatile cell system for transient gene expression analysis. Nat. Protoc. 2007, 2, 1565-1572. [CrossRef]

31. Bari, R.; Pant, B.D.; Stitt, M.; Scheible, W.-R. PHO2, MicroRNA399, and PHR1 Define a Phosphate-Signaling Pathway in Plants. Plant Physiol. 2006, 141, 988-999. [CrossRef] [PubMed]

32. Zhu, J.-K. Abiotic Stress Signaling and Responses in Plants. Cell 2016, 167, 313-324. [CrossRef] [PubMed]

33. Von Mering, C.; Jensen, L.J.; Snel, B.; Hooper, S.D.; Krupp, M.; Foglierini, M.; Jouffre, N.; Huynen, M.A.; Bork, P. STRING: Known and predicted protein-protein associations, integrated and transferred across organisms. Nucleic Acids Res. 2005, 33, D433-D437. [CrossRef]

34. Popescu, S.C.; Popescu, G.V.; Bachan, S.; Zhang, Z.; Seay, M.; Gerstein, M.; Snyder, M.; Dinesh-Kumar, S.P. Differential binding of calmodulin-related proteins to their targets revealed through high-density Arabidopsis protein microarrays. Proc. Natl. Acad. Sci. USA 2007, 104, 4730-4735. [CrossRef]

35. Zielinski, R.E. Camodulin and calmodulin-binding proteins in plants. Annu. Rev. Plant Physiol. Plant Mol. Biol. 1998, 49, 697-725. [CrossRef]

36. Adams, K.L.; Wendel, J.F. Polyploidy and genome evolution in plants. Curr. Opin. Plant Biol. 2005, 8, 135-141. [CrossRef]

37. Moore, R.C.; Purugganan, M.D. The evolutionary dynamics of plant duplicate genes. Curr. Opin. Plant Biol. 2005, 8, 122-128. [CrossRef] [PubMed]

38. Freeling, M. Bias in Plant Gene Content Following Different Sorts of Duplication: Tandem, Whole-Genome, Segmental, or by Transposition. Annu. Rev. Plant Biol. 2009, 60, 433-453. [CrossRef]

39. Mudgal, R.; Sandhya, S.; Chandra, N.; Srinivasan, N. De-DUFing the DUFs: Deciphering distant evolutionary relationships of Domains of Unknown Function using sensitive homology detection methods. Biol. Direct 2015, 10, 38. [CrossRef]

40. Grillet, L.; Lan, P.; Li, W.; Mokkapati, G.; Schmidt, W. IRON MAN is a ubiquitous family of peptides that control iron transport in plants. Nat. Plants 2018, 4, 953-963. [CrossRef] [PubMed]

41. Hirayama, T.; Lei, G.J.; Yamaji, N.; Nakagawa, N.; Ma, J.F. The Putative Peptide Gene FEP1 Regulates Iron Deficiency Re-sponse in Arabidopsis. Plant Cell Physiol. 2018, 59, 1739-1752. [CrossRef]

42. Rodríguez-Celma, J.; Pan, I.C.; Li, W.D.; Lan, P.D.; Buckhout, T.J.; Schmidt, W. The transcriptional response of Arabidopsis leaves to Fe deficiency. Front. Plant Sci. 2013, 4, 276. [CrossRef] [PubMed] 
43. Schaaf, G.; Honsbein, A.; Meda, A.R.; Kirchner, S.; Wipf, D.; von Wirén, N. AtIREG2 Encodes a Tonoplast Transport Protein Involved in Iron-dependent Nickel Detoxification in Arabidopsis thaliana Roots. J. Biol. Chem. 2006, 281, 25532-25540. [CrossRef] [PubMed]

44. Schuler, M.; Rellán-Álvarez, R.; Fink-Straube, C.; Abadia, J.; Bauer, P. Nicotianamine Functions in the Phloem-Based Transport of Iron to Sink Organs, in Pollen Development and Pollen Tube Growth in Arabidopsis. Plant Cell 2012, 24, 2380-2400. [CrossRef]

45. Yang, J.L.; Chen, W.W.; Chen, L.Q.; Qin, C.; Jin, C.W.; Chong, W.J.; Shi, Y.Z.; Zheng, S.J. The 14-3-3 protein GENERAL REGULATORY FACTOR11 (GRF11) acts downstream of nitric oxide to regulate iron acquisition in Arabidopsis thaliana. New Phytol. 2013, 197, 815-824. [CrossRef]

46. Kim, S.A.; LaCroix, I.S.; Gerber, S.; Guerinot, M.L. The iron deficiency response in Arabidopsis thaliana requires the phosphorylated transcription factor URI. Proc. Natl. Acad. Sci. USA 2019, 116, 24933-24942. [CrossRef] [PubMed]

47. Davletova, S.; Schlauch, K.; Coutu, J.; Mittler, R. The Zinc-Finger Protein Zat12 Plays a Central Role in Reactive Oxygen and Abiotic Stress Signaling in Arabidopsis. Plant Physiol. 2005, 139, 847-856. [CrossRef]

48. Mittler, R.; Kim, Y.; Song, L.; Coutu, J.; Coutu, A.; Ciftci-Yilmaz, S.; Lee, H.; Stevenson, B.; Zhu, J.-K. Gain- and loss-of-function mutations inZat10enhance the tolerance of plants to abiotic stress. FEBS Lett. 2006, 580, 6537-6542. [CrossRef]

49. Sun, J.; Jiang, H.; Xu, Y.; Li, H.; Wu, X.; Xie, Q.; Li, C. The CCCH-Type Zinc Finger Proteins AtSZF1 and AtSZF2 Regulate Salt Stress Responses in Arabidopsis. Plant Cell Physiol. 2007, 48, 1148-1158. [CrossRef] [PubMed]

50. Seo, P.J.; Kim, M.J.; Park, J.-Y.; Kim, S.-Y.; Jeon, J.; Lee, Y.-H.; Kim, J.; Park, C.-M. Cold activation of a plasma mem-brane-tethered NAC transcription factor induces a pathogen resistance response in Arabidopsis. Plant J. 2010, 61, 661-671. [CrossRef]

51. Yang, Z.-T.; Lu, S.-J.; Wang, M.-J.; Bi, D.-L.; Sun, L.; Zhou, S.-F.; Song, Z.-T.; Liu, J.-X. A plasma membrane-tethered transcription factor, NAC062/ANAC062/NTL6, mediates the unfolded protein response in Arabidopsis. Plant J. 2014, 79, 1033-1043. [CrossRef]

52. Bouché, N.; Yellin, A.; Snedden, W.A.; Fromm, H. Plant-specific calmodulin-binding proteins. Annu. Rev. Plant Biol. 2005, 56, 435-466. [CrossRef] [PubMed]

53. Oh, M.-H.; Kim, H.S.; Wu, X.; Clouse, S.D.; Zielinski, R.E.; Huber, S.C. Calcium/calmodulin inhibi-tion of the Arabidopsis BRASSINOSTEROID-INSENSITIVE 1 receptor kinase provides a possible link between calcium and brassinosteroid signalling. Biochem. J. 2012, 443, 515-523. [CrossRef]

54. Zhu, X.; Dunand, C.; Snedden, W.; Galaud, J.-P. CaM and CML emergence in the green lineage. Trends Plant Sci. 2015, 20, 483-489. [CrossRef] [PubMed]

55. Park, H.C.; Park, C.Y.; Koo, S.C.; Cheong, M.S.; Kim, K.E.; Kim, M.C.; Lim, C.O.; Lee, S.Y.; Yun, D.-J.; Chung, W.S. AtCML8, a calmodulin-like protein, differentially activating CaM-dependent enzymes in Arabidopsis thaliana. Plant Cell Rep. 2010, 29, 1297-1304. [CrossRef] [PubMed]

56. Magnan, F.; Ranty, B.; Charpenteau, M.; Sotta, B.; Galaud, J.-P.; Aldon, D. Mutations in AtCML9, a calmodulin-like protein fromArabidopsis thaliana, alter plant responses to abiotic stress and abscisic acid. Plant J. 2008, 56, 575-589. [CrossRef] [PubMed]

57. Cho, K.; Nguyen, H.T.K.; Kim, S.Y.; Shin, J.S.; Cho, D.H.; Hong, S.B.; Shin, J.S.; Ok, S.H. CML 10, a variant of calmodulin, modulates ascorbic acid synthesis. New Phytol. 2016, 209, 664-678. [CrossRef]

58. Leba, L.-J.; Cheval, C.; Ortiz-Martín, I.; Ranty, B.; Beuzón, C.R.; Galaud, J.-P.; Aldon, D. CML9, an Arabidopsis calmodulin-like protein, contributes to plant innate immunity through a flagellin-dependent signalling pathway. Plant J. 2012, 71, 976-989. [CrossRef] [PubMed]

59. Zhu, X.; Robe, E.; Jomat, L.; Aldon, D.; Mazars, C.; Galaud, J.-P. CML8, an Arabidopsis Calmodulin-Like Protein, Plays a Role inPseudomonas syringaePlant Immunity. Plant Cell Physiol. 2016, 58, 307-319. [CrossRef]

60. Yap, K.L.; Kim, J.; Truong, K.; Sherman, M.; Yuan, T.; Ikura, M. Calmodulin Target Database. J. Struct. Funct. Genom. 2000, 1, 8-14. [CrossRef]

61. Stothard, P. The Sequence Manipulation Suite: JavaScript Programs for Analyzing and Formatting Protein and DNA Se-quences. BioTechniques 2000, 28, 1102-1104. [CrossRef]

62. Hu, B.; Jin, J.; Guo, A.-Y.; Zhang, H.; Luo, J.; Gao, G. GSDS 2.0: An upgraded gene feature visualization server. Bioinformatics 2014, 31, 1296-1297. [CrossRef]

63. Drozdetskiy, A.; Cole, C.; Procter, J.; Barton, G.J. JPred4: A protein secondary structure prediction server. Nucleic Acids Res. 2015, 43, W389-W394. [CrossRef]

64. Wang, Y.; Tang, H.; DeBarry, J.; Tan, X.; Li, J.; Wang, X.; Lee, T.-H.; Jin, H.; Marler, B.; Guo, H.; et al. MCScanX: A toolkit for detection and evolutionary analysis of gene synteny and collinearity. Nucleic Acids Res. 2012, 40, e49. [CrossRef] [PubMed]

65. Lescot, M.; Déhais, P.; Thijs, G.; Marchal, K.; Moreau, Y.; Van de Peer, Y.; Rouzé, P.; Rombauts, S. PlantCARE, a database of plant cis-acting regulatory elements and a portal to tools for in silico analysis of promoter sequences. Nucleic Acids Res. 2002, 30, 325-327. [CrossRef]

66. Ying, S.; Zhang, D.-F.; Fu, J.; Shi, Y.-S.; Song, Y.-C.; Wang, T.-Y.; Li, Y. Cloning and characterization of a maize bZIP tran-scription factor, ZmbZIP72, confers drought and salt tolerance in transgenic Arabidopsis. Planta 2012, 235, 253-266. [CrossRef] [PubMed]

67. Brandão, M.M.; Dantas, L.L.; Silva-Filho, M.C. AtPIN: Arabidopsis thaliana Protein Interaction Network. BMC Bioinform. 2009, 10, 454. [CrossRef] [PubMed]

68. Consortium, A.I.M. Evidence for Network Evolution in an Arabidopsis Interactome Map. Science 2011, 333, $601-607$. 